\title{
Towards Context-Aware User Modeling
}

\author{
Michael Samulowitz \\ Corporate Technology \\ Siemens AG \\ Otto-Hahn-Ring 6 \\ D-81730 Munich, Germany \\ Michael.Samulowitz@mchp.siemens.de
}

\begin{abstract}
Research in Ubiquitous and Wearable Computing yielded several types of context-aware applications, dramatically changing the way to interact with computers. Personalizing these applications is a key concern; therefore we investigate ways to apply user modeling to the paradigm of context-aware systems. Our initial work proposes (1) a hierarchy that relates user model data to contextual information, and (2) operations for manipulating the hierarchy. Deploying the operations the hierarchy can act as a substrate for communicating the user's wishes concerning system behavior.
\end{abstract}

\section{Introduction}

Context-Aware applications [1] adapt to the context (e.g. location or time) of use. Especially, work in ubiquitous [2] and wearable computing [3,4] substantiates the deployment of context-aware applications (or services). In these settings, the user has increased freedom of mobility. The increase of mobility creates situations where the user's context, such as location of a user and the people and objects around her, is more dynamic. Both wearable and ubiquitous computing have given users the expectation that they can access services whenever and wherever they are [5]. These services should adapt to the wide range of possible user situations. Therefore the concept of context is a useful method, providing means to determine the user's situation, which may be used to tailor the systems behavior accordingly.

Context-aware applications principally aim to improve human-computer interaction by implicitly using context information. A further improvement can be accomplished by personalizing these applications. Generally, systems providing personalized applications (or services) are based on a user model. A user model contains the system's assumptions about the user, which are utilized to adapt to the user's needs [6].

Here, we want to investigate approaches for user modeling suited to context-aware systems, taking into account their special requirements. 


\section{Basics on Context}

The notion of context has significant impact on application design for mobile users, where context is subject to constant change. If context is accessible to applications its possible to dynamically adjust behavior to the current user's context. Prior to discussing context-aware applications and their personalization, it is helpful to provide a definition for context.

Here we render a definition given in [5], which refines and generalizes several definitions that can be found in other sources, e.g. [1,7]:

Context is any information that can be used to characterize the situation of an entity. An entity is a person, place, or object that is considered relevant to the interaction between a user and an application, including the user and applications themselves.

Context information can be categorized in different types of information; certain types of context information are more important than others, particularly considering practical use. These are location, identity, activity, and time. These types of context are designated as primary context. Secondary context types may be derived by given primary context information. For example, given a person's identity (primary context) it may be possible to acquire its phone number (secondary context).

In addition [5] gives a definition for a context-aware system:

A system is context-aware if it uses context to provide relevant information and/or services to the user, where relevancy depends on the user's task.

A rich set of usage scenarios typically enabled by context-aware systems can be found in literature; for example mobile tour guides [8,9], or context-based retrieval applications that gather and store context information and allow later information retrieval based on context information [10].

\section{Basics on User Modeling}

User modeling is concerned with how to represent the user's knowledge and interaction within a system to adapt those systems to the needs of users [11]. Work in user modeling is tackled by several research fields, including artificial intelligence, human-computer interaction, psychology and education, which investigate ways to construct, maintain and exploit user models. The following list states general examples where user models obviously enhance usability of applications by embedding user related information:

- Message filtering [12]

- Adaption of user interfaces

- Personalized web pages

- $\quad$ Computer-based training (CBT) 
Generally, the following matters are key concerns to user model management: (1) gathering relevant data, (2) appropriate representation schemes, and (3) consistency of user model data. The management of user models is typically done by agents, which constantly monitor user-system interaction, e.g. [15].

\section{The Challenge: User Modeling and Context-Awareness}

The context-aware computing paradigm helps to improve user-system interaction by implicitly considering contextual information. Personalization deploying user modeling is an another strategy to ease interaction. We propose a way to combine both strategies. Therefore our work aims to develop schemes for user modeling, taking into account contextual information. In particular, we propose a scheme that allows associating user model data to certain context types. For example, we could associate user model data to the user's visited locations (context type: location); hence, it would be possible to design context-aware applications which adapt their behavior to both, the current location and the associated user model data for this place. Besides considering context-awareness our user modeling scheme should regard the fact that a mobile user potentially uses multiple applications; the following list states examples of possibly context-aware applications where user models enhance usability:

- Mobile e-commerce

- Location aware services

- Environment configuration [13]

- Process activation/deactivation in dependence of context [14]

The listed examples clarify the difficulty to introduce a single user model for contextaware systems, because eventual personalization covers several areas of use and there is no common model that fits to all of them. For this reason we propose to separate concerns in user modeling for context-aware systems. This would result in multiple user models each being specialized on a certain context of use. Still there is information common to all of them (or at least to a sub-set) e.g. superior settings regarding security or privacy issues, this information should be propagated to the other user models, in order to attain consistent system behavior.

\section{Discussion: A Hierarchy of User Models}

As an appropriate modeling scheme we propose a hierarchy of user models in order to organize user-centric data in context-aware systems. In correspondence to the requirements outlined in the previous paragraph the scheme should allow to model data considering context types and common data structures, that may be communicated among different user models. 
The initial design for our modeling scheme provides user model entities, and associations between them. Entities may present the user model data for a certain application, or they may represent user specific data related to context information. Associations relate entities to each other by indicating common data structures between entities, or they are used for assigning user related data to particular context information. The following two examples illustrate the main principles.

Figure 1 states an example for communicating common data structures between user models. The figure shows three user model entities (depicted by circles). The PeopleFinder and the Mobile e-commerce entity are associated to an entity representing the user's preferred policies regarding security and privacy. Hence, if a user changes these policies, information about this should be propagated to the user model entities associated (PeopleFinder and Mobile e-commerce). If the user selects a "high" privacy level he should neither being locatable nor being annoyed by offers to buy.

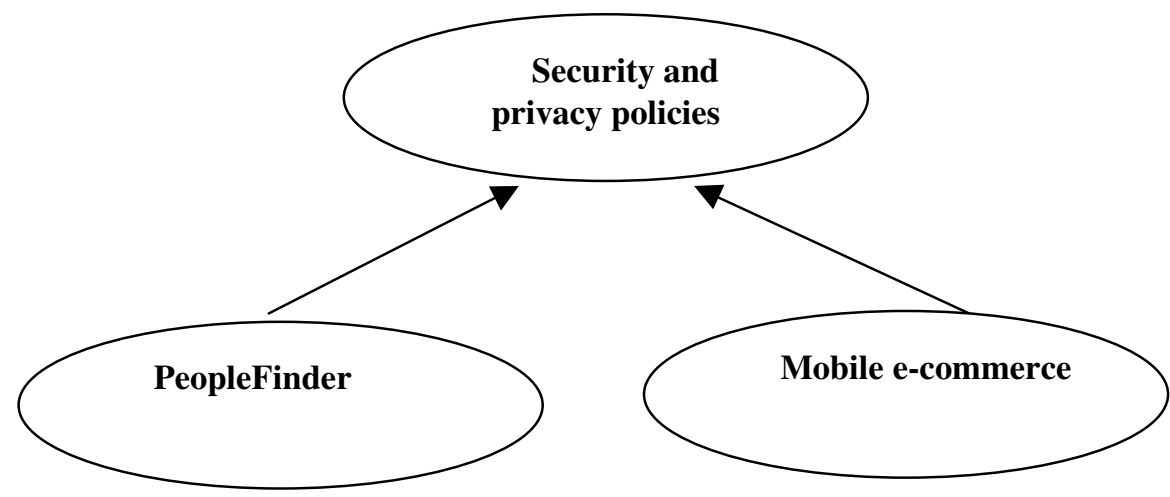

Figure 1. User Models related to each other

Figure 2 depicts another example for distributing user model data on a hierarchy of entities, each entity representing a certain subset of the user model data. In particular, this example focuses on relating user-centric data to context information (location); it takes in account spatial concerns and provides a higher degree of granularity in comparison to the first example. The left side of the figure (Room A) shows the user model data associated to a room; it mainly includes processes which are triggered by certain user actions [14], and references to resources the user normally uses. In this example, the sphere of action of the entities processes and resources is constrained by location (Room A). If the same user wants to have the same settings ("look \& feel") in room $\mathrm{B}$ as in room $\mathrm{A}$, he would ask the system to do so. The system would automatically transfer all settings related to room A (including the associated entities) to room B. (The set of candidates for transfer operation could be determined by referential integrity constraints.)

Transfer is not always trivial; in the given example it is not sure that room B is equally equipped (e.g. computing resources) as room A is. Hence, this could lead to partial transfer or intelligently adapting functions. 


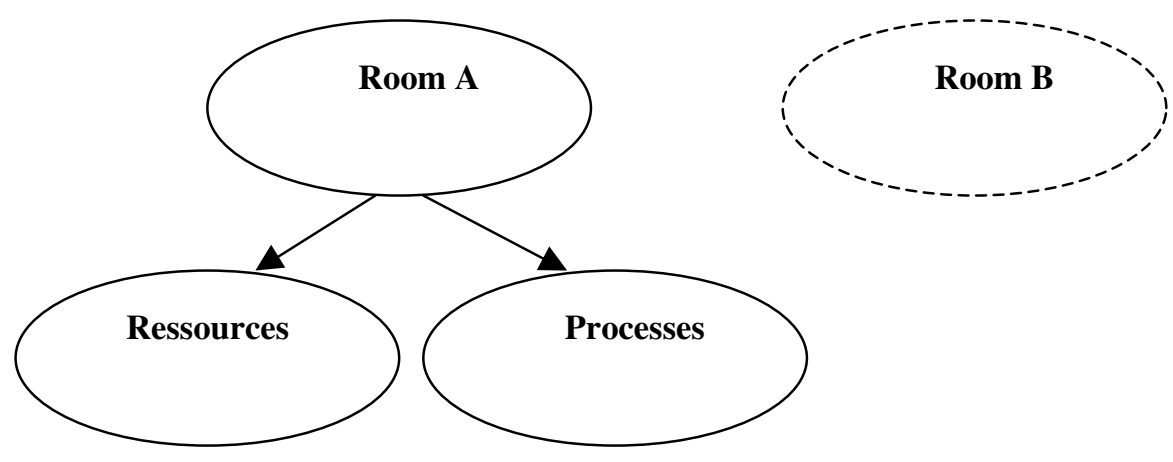

Figure 2. User Model Data and Context

The previous examples introduced two operations on the hierarchy: propagation and transfer. In order to implement these operations and probably others, we could associate an agent to each of the entities. These agents could co-operatively implement transfer and propagate operations.

\section{Summary}

We presented a novel approach to user modeling considering contextual information. Our future work seeks to improve our modeling scheme, especially regarding the following issues: (1) defining a general modeling scheme (for the moment it is basically introduced by examples), (2) how applications can actually evaluate information stored in the hierarchy, (3) proposing scenarios that consider other context types (e.g. activity or time).

\section{References}

1. Schilit, B.N., Adams, N.I., Want, R. Context-aware computing applications. Proceedings of the Workshop on Mobile Computing Systems and Applications, pp 85-90. IEEE Computer Society, Santa Cruz, CA, 1994

2. Weiser, M. Some Computer science issues in ubiquitous computing. Communications of the ACM 36, 7 (July 1993), 75-83

3. T. Starner, S. Mann and B. Rhodes The MIT wearable computing web page. http://wearables.www.media.mit.edu/projects/wearables.

4. Abowd, G. D., A. K. Dey, R. Orr, and J. Brotherton, "Context-awareness in Wearable and Ubiquitous Computing,” GVE, Technical Report GIT-GVU-97-11, May 1997.

5. Dey, A.K. \& Abowd, G.D. Towards a better understanding of context and context-awareness. GVU Technical Report GIT-GVU-99-22, College of Computing, Georgia Institute of Technology, 1999 
6. Alfred Kobsa. User modeling and user-adapted interaction. Proceedings of the CHI'94 conference companion on Human factors in computing systems, 1994, 415 - 416

7. Pascoe, J. Adding Generic Contextual Capabilities to Wearable Computers. 2nd International Symposium on Wearable Computers, 146-153, 1998

8. Lancaster University. The Active Badge Tourist Application. Available at http://www.comp.lancs.ac.uk/computing/research/mpg/most/abta_project.html.

9. Abowd, G.D., Atkeson, C.G., Hong, J., Long, S., Kooper, R., and Pinkerton, M. Cyberguide: A Mobile Context-Aware Tour Guide. ACM Wireless Networks 3, 421-433.

10. Rhodes, B. J. The Wearable Remembrance Agent, in Proceedings of the 1st International Symposium on Wearable Computers, ISWC ' 97 (Cambridge MA, October 1997), IEEE Press, 123-128.

11. M. Brown, Eugene Santos, Sheila B. Banks and Mark E. Oxley. Using explicit requirements and metrics for interface agent user model correction. Proceedings of the second international conference on Autonomous agents , 1998, Pages $1-7$

12. M. Matthew, C. Schmandt. CLUES: dynamic personalized message filtering. Proceedings of the ACM 1996 conference on Computer supported cooperative work, 1996, pages 113 121

13. A. Pentland, "Smart Rooms", Scientific American, April 1993

14. R. Want, A. Hopper, V. Falcao, and J. Gibbons. The active badge location system. ACM Trans. On Info. Sys., vol. 10, no.1, pp. 91-102, January 1992

15. Rob Barrett, Paul P. Maglio and Daniel C. Kellem. How to personalize the Web. Conference Proceedings on Human factors in computing systems, 1997, pages 75 - 82 